\title{
Soziale Dimensionen von Smart Metering am Arbeitsplatz
}

\author{
Matthias Betz, Tobias Schwartr. \\ Fraunhofer FIT, Sankt Augustin
}

\section{Einleitung}

Bei wachsendem globalen Energiebedarf und einer zunehmender Verknappung der natürlichen Ressourcen, ist die Zahl von Unternehmen, die den betrieblichen Energiekonsum auf Einsparungspotenziale prüfen und gleichzeitig die Schaffung von nachhaltig energieeffizienten Arbeitsplätzen vorantreiben verhältnismäßig gering. Eine von der Deutschen Energie-Agentur (dena) in Auftrag gegeben Studie aus dem Jahre 2007 zur Aufdeckung von Hemmissen für Energieeffizienz in Unternehmen kommt zu dem Schluss, dass vor allem Fragen hinsichtlich der Finanzierung, Unklarheiten bei der der Erkennung von Ansatzpunkten zur technischen Umsetzung und zeitliche Problem Hürden in der unternehmerischen Praxis darstellen (dena 2007).

Die effiziente Nutzung der Energieressource Elektrizität im betrieblichen Alltag ist dabei häufig der mangelnden Transparenz des Verbrauchs, der resultierenden Kosten und der Wahrnehmung von Effizienzpotenzialen geschuldet. Bleibt der tatsächliche Energiekonsum diffus ist eine Bewertung und Optimierung des Ressourceneinsatzes ein diffiziles Unterfangen. Verschiedene Lösungsansätze zielen dabei darauf ab, durch die Verwendung von intelligenter Messtechnologie notwendige Energietransparenz zu schaffen und dem Benutzer auf seine Bedürfnisse und Kompetenzen abgestimmte Kontrollmöglichkeiten an die Hand zu geben. Durch die detaillierte Erfassung aller relevanten Verbrauchsdaten werden Informationen für die Identifikation von Einsparungspotenzialen und zur Erstellung einer persönlichen Energiebilanz gewonnen. Vermehrt werden hierbei innovative Informations- und Gebäudesystemtechnologien gekoppelt und so neuartige Strategien zur effizienten Energienutzung erschlossen. Energiemanagementsysteme (EMS) stellen dabei eine Softwaretechnische Unterstützung dar, welche Verbrauchsinformationen zur Aufdeckung von Einsparpotenzialen für den Nutzer angemessen visualisieren, um neue Kompetenzen und Handlungsspielräume für einen effizienten Umgang mit Elektrizität zu erschließen. Softwaresysteme zur Steuerung von ,intelligenter Messtechnologie am Arbeitsplatz' stellen hierbei ein 
neues Anwendungsfeld dar, deren Einsatz und deren Einfluss auf das soziale System ,Arbeitsplatz' sind bisher jedoch nur unzureichend erforscht.

Betrachtet man den Markt für intelligente Messtechnologie, wurden mit der Einführung der $\$ \int 21 \mathrm{~b}$ und 40 EnWG sowie der Messzugangsverordnung im Oktober 2008 vorherrschenden Rahmenbedingungen für das Mess- und Zählwesen in erheblichem Umfang neu geregelt. Für Unternehmen mit einem Energieverbrauch von über $100.000 \mathrm{kWh}$ pro Jahr ist diese Vorgabe eine Lastgang- und Leistungsmessung durchzuführen bereits heute bindend. Die dabei gewonnenen Informationen über den Energiekonsum dienen aber nicht der Illustration von Verbräuchen und Schaffung von Verbrauchstransparenz auf Ebene der Arbeitspraxis, sondern vielmehr werden die Informationen zur betrieblichen Planung herangezogen. Obwohl sich bisher der überwiegende Teil gesetzgeberischer Maßnahmen auf den Bereich der privaten Haushalte beziehen, sind die damit verfolgten Ansätze - die Schaffung von Transparenz - ein erklärtes Ziel, dass auch für den energieeffizienten Büroarbeitsplatz maßgebend und relevant ist.

Zusätzlich tragen Produktinnovationen im Bereich der intelligenten Messtechnologien zu einer verbreiteten Nutzung im betrieblichen Anwendungskontext bei. Vor diesem Hintergrund gewinnt die Gestaltung energieeffizienter Büroarbeitsplätze und eines verantwortungsvollen Ressourcenmanagement mittels Smart Metering zunehmend an Bedeutung. Im Sinne eines soziotechnischen Gestaltungsverständnisses müssen aber auch soziale Fragestellungen bei der Implementierung und Ausgestaltung solcher Feedbacksysteme berücksichtigt werden. Dieser Bereich der sozialen Dimension von SmartMetering ist jedoch bisher nur unzureichend erforscht. Der vorliegende Beitrag beschreibt die Ergebnisse einer qualitativen Studie zu sozialen Aspekten von SmartMetering am Arbeitsplatz. Es wird aufgezeigt, wie Mitarbeiter anhand von SmartMetering-Informationen ein Problembewusstsein zum Thema ,Energieeffizienter Arbeitsplatz aufbauen und gemeinsam Lösungsstrategien aushandeln. Zusätzlich illustriert die Studie, wie intelligente Messtechnologien im sozio-technischen Anwendungsfeld ,Arbeitsplatz wahrgenommen werden und welche Aussagekraft detaillierte Stromverbrauchsdaten in Hinblick auf energieeffizientes Verhalten am Arbeitsplatz haben.

Der Beitrag ist wie folgt strukturiert: Zuerst werden relevante Ansätze, verwandte Themen und Technologien in dem hier adressierten Arbeitsbereich betrachtet. Es folgt die verwendete Methodik und das Vorgehen bei der Umsetzung der Studie. Im Anschluss zeigen wir anhand des empirischen Materials, wie in einem Gruppen-Auswertungsworkshop mittels SmartMetering-Messdaten gemeinschaftlich Möglichkeiten für mehr Energieeffizienz am Arbeitsplatz ausgehandelt wurden und welche Effekte sich im Nachgang des Workshops bezüglich des Energieverbrauchs am Arbeitsplatz eingestellt haben. Die Phänomene auf der sozialen Ebene werden dabei mit Messdaten des Energieverbrauchs vor, unmittelbar nach und in einer längerfristigen Perspektive nach dem veranstalteten Workshop in Relation gesetzt, um die entstehenden Effekte hinsichtlich des tatsächlichen Verbrauchs nachvollziehbar zu machen. 


\section{Stand der Forschung und verwandte Arbeiten}

Eine zentrale Herausforderung bei der Gestaltung nachhaltig energieeffizienter Büroarbeitsplätze ist der bewusste Einsatz der zentralen Energie-Ressource Elektrizität. Allein 16 Millionen Erwerbstätige arbeiten in Deutschland an einem Büroarbeitsplatz. In den letzten 50 Jahren ist der Anteil der in Büros Beschäftigten an der Erwerbsbevölkerung von etwa 10 Prozent auf knapp 50 Prozent angestiegen (Hall 2007). Die rasante elektronische Entwicklung in den letzten 20 Jahren hat im gleichen Zeitraum das Büro als Arbeitsplatz strukturell verändert. Im Zeitalter der digitalen Datenverarbeitung ist die Anzahl von Büroarbeitsplätzen ohne elektrische Geräte (z. B.: Computer, Drucker, Telefon) verschwindend gering. Klassische Arbeitswerkzeuge wie Ordner, Papier und Stift wirken dabei oftmals schon antiquiert.

Studien zur Ressourcenverwendung in Verwaltungs- und Bürogebäuden zeigen, dass sich der Energieverbrauch allgemein fast gleichmäßig auf die Faktoren Elektrizität und Wärme aufteilt. Bezogen auf den gesamten Energieverbrauch eines Unternehmens (mit Ausnahme des produzierenden Gewerbes) entfällt ein Anteil von ca. $30 \%$ auf die durch Bürogeräte entstehenden Stromkosten.

Untersuchungen markieren, dass gerade im Bereich des Büroarbeitsplatzes hohe Einsparpotenziale verborgen liegen. Wer sein Büro mit energieeffizienten Infrastruktur und Geräten ausstattet, kann im Vergleich zu einem ineffizient ausgestatteten Arbeitsplatz bis zu 75 Prozent der Stromkosten einsparen (dena 2007a). Dennoch verzichten nach wie vor viele Unternehmen auf Energieeffizienzmaßnahmen. Als Gründe dafür werden die Sicherstellung der Finanzierung und fehlende Sachkenntnisse genannt. Dies ist das Ergebnis einer Umfrage, die die Deutsche Energie-Agentur (dena) im Rahmen der Initiative EnergieEffizienz bei Unternehmen verschiedener Branchen durchgeführt hat. 36 Prozent der befragten Unternehmen, die bislang keine Schritte zur Steigerung der Energieeffizienz eingeleitet haben, sehen in der Finanzierung von Energieeffizienzmaßnahmen die größte Herausforderung. 32 Prozent der Unternehmen verfügen nach eigenen Angaben nicht über genügend Informationen, um sich der Energieeffizienzsteigerung widmen zu können (dena 2007b).

Möchte man den Büroarbeitsplatz nachhaltig energieeffizienter gestalten und verborgenen Einsparpotenziale heben, gilt es die vorherrschenden Dynamiken dieses Anwendungsfeld bei der Gestaltung von unterstützenden Assistenzsystemen mit zu berücksichtigen. Dabei ist die Verwendung einer energieeffizienten Infrastruktur und die richtige Konfiguration der am Arbeitsplatz verwendeten Geräte ein wichtiger Beitrag zur Erreichung dieses Ziels. Feedback-Systeme und intelligente Messtechnologie stellen aber gleichermaßen bedeutende Instrumente zur Umsetzung von Klimaschutzmaßnahmen dar. Aktuelle internationale Forschungsbeiträge zeigen in diesem Zusammenhang auf, dass die Erhöhung der Transparenz durch zeitnahes Feedback und die Zurechenbarkeit des Verbrauchs zu einzelnen Geräten wesentliche Faktoren darstellen und meist einer tiefergehenden Bewertung 
des Stromverbrauchs vorgelagert sind bzw. diese erst ermöglichen (Chetty et al. 2009; Chetty et al. 2008; Darby 2006; Darby 2001; Dillahunt et al. 2009).

\section{Methodologie}

Um die Effekte durch Verbrauchsinformationen und zeitnahes Feedback über den Energiekonsum am Arbeitsplatz zu erforschen, wurden in einer öffentlichen Einrichtung mit mehr als 950 Mitarbeitern zwei Büroräume mit intelligenter Messtechnologie ausgestattet. Diese ermöglichte es, den Gesamtverbrauch dieser Räume zu erfassen. In den Büroräumen sind sieben Standardbüroarbeitsplätze vorhanden. Im Sinne eines aktionsforscherischen Vorgehens, wurden die Mitarbeiter mit den gemessenen Daten im Rahmen von einer Auswertungssitzung konfrontiert. Der Auswertungsworkshop wurde aufgezeichnet und im Nachgang qualitativ ausgewertet. Die Räume wurden nach den Kriterien der technischen Machbarkeit ausgewählt. Die Messdaten wurden dabei über Sensoren im Sicherungskasten abgegriffen und mittels Funk an einen zentralen Datensammler übertragen, der im Anschluss zu Auswertungszwecken ausgelesen wurde. Die Messdaten wurden dabei für die Gruppenworkshops aufbereitet und visualisiert. Die Messreihen der Verbrauchsmessung lagen in einer Granularität „Verbrauch in kWh in 5 Minuten“ vor, d.h. die Sensoren lieferten immer den Stromverbrauch der letzten 5 Minuten an den zentralen Datensammler.

Zunächst wurden die Mitarbeiter in den Büroräumen gefragt, ob sie mit der Messung des Stromverbrauchs in ihren Räumen einverstanden seien. Die Mitarbeiter wussten zu Beginn der Studie weder in welchem Umfang, noch mit welcher Technologie die Messungen durchgeführt wurden bzw. welche Form das Ergebnis der Messung haben wird. Zwischen der Einholung des Einverständnisse und der Installation der Messtechnologie verging einige Zeit. Nach der Installation der Messtechnik wurde der Lastgang der Büroräume drei Wochen lang aufgezeichnet. Nach Ablauf dieser Zeit wurde der gemeinsame Auswertungs- und Analyseworkshop mit den betreffenden Mitarbeitern angesetzt. Zur Durchführung wurde der erfasste Lastgang graphisch als Liniendiagramm aufbereitet. Dabei handelte es sich konkret um die Darstellung des Gesamtstromverbrauchs der Büroräume über die Zeit (Lastgang) der Messdauer von drei Wochen, wobei eine partielle Skalierung bis auf Fünfminuteneben an jedem gewünschten Zeitpunkt möglich war. Aus Gründen der Übersichtlichkeit wurde als Einstiegsdarstellung der graphisch dargestellte Lastgang über den gesamten Messzeitraum gewählt (vgl. Abbildung 1). Die gemeinsame Diskussion des Lastgangs im Workshop dauerte insgesamt 115 Minuten. Nach dem Workshop wurden den Mitarbeitern in den Büroräumen zusätzlich Sensoren ausgehändigt, um den aktuellen tatsächlichen Stromverbrauch von elektrischen Geräten explorativ ermitteln zu können. Es handelte sich dabei um einfache Messuhren zur Messung der Aufnahmeleistung, wie sie kostengünstig im Elektrohandel erwerblich sind. Der Wunsch nach solchen Kontrollmöglichkeiten 
hat sich im Rahmen der Gruppendiskussion selbstständig herausgebildet. Die Messuhren konnten die Mitarbeiter nach Belieben in den Büroräumen zwischen den Geräten und den vorhandenen Steckdosen anbringen. Die Mitarbeiter taten dies mit der Motivation, Verbräuche besser abschätzen und zuordnen zu können. Die Messung des Gesamtstromverbrauchs der Räume wurde dabei weiter fortgeführt.

\section{Auswertung und Analyse der Studie}

In der Analyse präsentieren wir Ergebnisse der durchgeführten qualitativen Studie, welche die soziale Auswirkung von intelligenter Messtechnologie in Unternehmen erforscht. Untersuchungsinteresse war es, soziale Aspekte im Zusammenhang mit intelligenter Messtechnologie näher zu beleuchten und zu überprüfen, welche Nebeneffekte die neu gewonnen Transparenz über den Stromverbrauch auf das soziale System einer Organisation hat. Das empirische Material wurde daraufhin untersucht, welche sozialen Interdependenzen sich innerhalb einer Gruppe von Mitarbeitern durch die Anwendung intelligenter Messtechnologie bei Büroarbeitsplätzen ergeben.

\subsection{Auswertungsworkshop}

Im Folgenden wird nun der Ablauf des Workshops basierend auf der Messung des Lastgangs des Zeitraums von drei Wochen vor dem Workshop dargestellt und die darin beobachteten Phänomene näher untersucht. An dem Workshop nahmen insgesamt sechs Personen teil. Vier der sechs Personen (davon eine Frau) arbeiten in den gemessenen Büroräumen und haben bis zu diesem Zeitpunkt keinen Zugang zu den Messdaten. Nach einer kurzen Einführungsphase, in der den Teilnehmern des Workshops nochmals das gesamte Setting erläutert wurde, wurde den Anwesenden über einen Beamer eine graphische Visualisierung der Verbrauchsdaten präsentiert (vgl. Abbildung 1). In diesem Zusammenhang wurden die Einheiten und die Skalierung der Daten kurz erläutert und offene Fragen geklärt. Dabei stellte sich heraus, dass den Teilnehmern die Einheit ,kWh' geläufig war, eine Darstellung des Verbrauchs in 5-Minuten Abschnitten in Form eines Lastgangs jedoch zunächst schwer verständlich erschien. Vor allem die auf die kurzen Intervalle verteilten Verbräuche vielen den Teilnehmern als sehr gering auf, was eine Erläuterung seitens der Workshop-Leitung erforderte. Ein Hochrechnen auf Verbräuche pro Stunde erleichterte das Verständnis der Einheit , $\mathrm{kWh}$ ' und machte die angegebene Messwerte vergleichbar mit privaten Verbräuchen.

Bereits in den ersten 12 Minuten des Workshops wurden, nach näherer Betrachtung des Lastgangs, die Verbräuche auf typische Verhaltensmuster hin reflektiert. So fällt einem Teilnehmer auf, dass in den Mittagsstunden der Verbrauch etwas zurückgeht, was in der Gruppe daraufhin diskutiert und gedeutet wird. Man kommt zu dem Schluss, dass durch die Abwesenheit und Inaktivität an den Com- 
putern sich die Monitore nach einiger Zeit automatisch ausschalten. Dies ist ein erster Hinweis darauf, dass die Verbrauchsdaten für die Workshopteilnehmer an Bedeutung gewinnen, wenn sie die Messdaten und Lastgangverläufe in Relation mit typischen Verhaltensmustern setzen.

Unmittelbar danach fällt einem anderen Teilnehmer auf, dass die Verbräuche außerhalb der Arbeitszeiten (7:30-19:30 Uhr; in Abbildung 1 bis Abbildung 3 hellgrau eingefärbt) seiner Einschätzung nach sehr hoch ausfallen. Er bezeichnet diesen Verbrauch im Folgenden als „Grundlast“. Ohne die Zahlen genau zu kennen, sagt er im Diskussionsverlauf wörtlich:

A: „Aber ist die Grundlast nicht unglaublich hoch? Die ist ja [pause] 50\% unseres Bedarfs ist ja Grundlast! [...] Das heißt, wir verbrauchen eigentlich gar nicht so viel, eigentlich. Irgendwie gut, eigentlich. Versteht ibr? [kichert]".

Dieser Einwurf wird von allen Teilnehmern aufgegriffen und es folgt eine Diskussion über die mögliche Zusammensetzung der "Grundlast". Es werden Netzteile erwähnt, die eingesteckt bleiben, auch wenn die Geräte daran nicht genutzt oder ausgeschaltet sind, PCs, die nach Feierabend nicht heruntergefahren werden, Akkuladegeräte, eine gemeinsam genutzte Stereoanlage, lokal betriebene Entwicklungsserver (es handelt sich dabei in der Regel um alte, nicht mehr benötigte Desktop-PCs), ein Smartboard usw. Die Teilnehmer stellen gemeinsam fest, dass die Grundlast an einigen Wochenenden etwas niedriger ausfällt, was auf heruntergefahrene PCs und die ausgeschaltete Stereoanlage zurückgeführt wird. Ob diese Vermutungen stimmen, kann in dem Workshop nicht geprüft werden. Obwohl die Teilnehmer des Workshops keinerlei persönliche Konsequenzen für den Strom verbrauch zu tragen haben, weder finanziell, wie dies im privaten Bereich der Fall

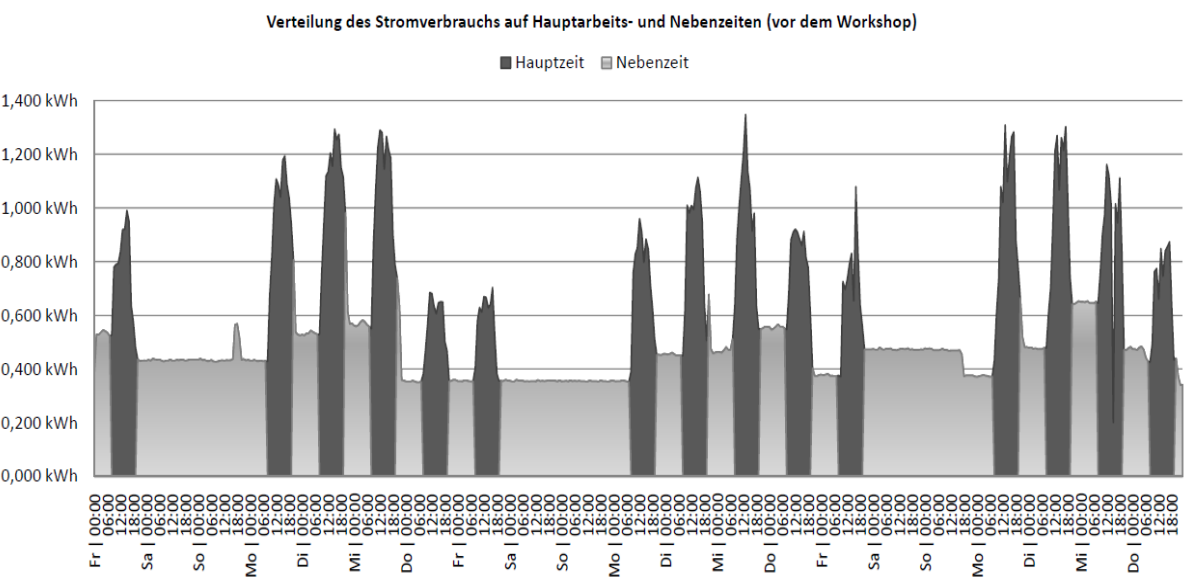

Abbildung1: Lastgang Zeitraum: 3 Wochen vor dem partizipativen Auswertungsworkshop 
sein würde, noch im Rahmen anderer Sanktionen, wird das Nicht-Ausschalten von Geräten außerhalb der Arbeitszeiten als Verschwendung wahrgenommen. Dies wird unter anderem an folgenden Gesprächsverlauf deutlich.

A: „Also den Verstärker... Also wenn ich Morgens komme, und ich sehe, dass der Verstärker noch an ist, hab ich immer ein schlechtes Gewissen. Weil wir ihn nicht ausgemacht baben. [kurze Pause] Also ich mache ibn schon auch aus. Auch bewusst. Weil ich auch da sitze."

B: „Den Verstärker mache ich auch immer aus. Also wenn ich letəter bin, dann mache ich den Verstärker aus. "

C: „Also ich nehm' das gar nicht wahr, weil das nicht mein Bereich ist, hinter dem Schreibtisch. Auf der anderen Seite.

B: „Du benut々t den Verstärker auch nicht... “

Im weiteren Verlauf versucht Teilnehmer A den Verbrauchsanstieg, den er persönlich verursacht, aus dem Lastgang herauszulesen, da er an einem bestimmten Tag schon sehr früh (7:00 Uhr) zur Arbeit gekommen, und zu dieser Zeit noch niemand anwesend gewesen sei. Nach längerem Suchen kann der spezifische Anstieg in den Morgenstunden identifiziert werden.

Im Laufe der weiteren Diskussion des Lastgangs werden weitere Muster erkannt und besprochen. So fällt beispielsweise auf, dass am ersten Wochenende des Messzeitraums eine höhere Grundlast zu verzeichnen ist, als am darauf folgenden Wochenende. Man versucht daraufhin gemeinsam herauszufinden, was genau der Grund für dieses unterschiedliche Niveau sein könnte. Zunächst wird vermutet, dass der Desktop von Teilnehmer B heruntergefahren wurde. Diese Vermutung kann jedoch von den Teilnehmern anhand des Lastgangs nicht eindeutig nachvollzogen werden.

Es wird nun darüber diskutiert, welcher Verbrauch welchem Kollegen zugerechnet werden kann. Ein Teilnehmer (C) stellt etwas provokativ, jedoch freundschaftlich kollegial die Frage:

C: „Wann sprechen wir eigentlich darüber, wer schuldig ist für das Ganze? [...] Ich glaube

$B$ ist schuld. Das ist dem total egal, das mit dem Strom."

Workshop-Leiter: „W arum glaubst du das?"

C: „Weil alles eingesteckt wird, und wenn einmal was eingesteckt ist, dann wird es nie wieder rausgesteckt. Das ist die Grundregel. "

A: „Wir haben uns auch schon mal darüber unterhalten. Irgendwann hast Du oder ich oder so mal gesagt: ,Mensch B, mach doch Deinen Rechner mal am Wochenende aus. "

B: „Ich benutze den manchmal am Wochenende zu Hause. Zum anmelden, am Remotedesktop. Das ist ein Server. " [...]

A: Wenn Du so einen Knopf hättest: „Server jetzt bitte einschalten, wäre das ja auch ok.."

C: "Das wäre super, ja."

A: "Ja, dann könnte der aus sein."

C: „Das akzeptiere ich, das wäre ne gute Idee." 
Im weiteren Verlauf gehen die Teilnehmer Schritt für Schritt alle Geräte durch, die entweder nur von jeweils einzelnen Personen genutzt werden und welche maximalen Aufnahmeleistungen auf den Typenschildern der Geräte angegeben sind. Jeder der Teilnehmer neigt jedoch dazu so zu argumentieren, dass die persönlich genutzten Geräte notwendig sind, um produktiv arbeiten zu können. Es wird intensiv darüber diskutiert, ob der Stromverbrauch personenbezogen vergleichbar ist. In dieser Frage gehen die Positionen von Teilnehmer A und B kontrovers auseinander. Im weiteren Diskussionsverlauf verschiebt sich der thematische Fokus auf die "Grundlast" der gemeinsam verwendeten Geräte wie File-Server, Smartboard, Stereoanlage, und auf den Stromverbrauch bei Abwesenheit in den Nebenzeiten, der in dem hier vorgestellten Fall ca. 50\% des gesamten Stromverbrauchs in dem gemessenen Zeitraum ausmacht. Betrachtet man den Lastgang in Abbildung 1 fühlen sich alle Teilnehmer herausgefordert, die graugefärbten Bereiche zu minimieren. Man ist sich darüber einig, dass unabhängig vom tatsächlichen finanziellen Vorteil für die Einrichtung dieser Verbrauch unnötig sei. Auch geht die Argumentation weg von einer monetären hin zu einer Umwelt- und Klimamotivation:

C: „Aber wenn man mal überlegt, wie viel der Strom für so ne Einricbtung kostet... Die Einsparungen... Irgendwie enttäuschend. Strom ist einfach so billig! Das ist ja als ob man am Toilettenpapier sparen würde, weißt Du?."

A: „Kann man denn sagen, wie viel das in $\mathrm{CO}_{2}$ ist?

B: „Aber es geht ja um Optimierung. Die Frage ist doch: wie hoch ist das Optimierungspotential? [pause] Aber was ist das Optimierungspotential? Wenn man hier in der Kurve $30 €$ hat, und man kann das senken, so auf $24 €$ [pause] Interessiert mich nicht."

A: "Also, ich muss jetzt nicht unbedingt sehen, wie viel Geld mich das kostet. Wenn ich wüsste, dass der Verstärker superviel ausmacht, dann würd ich den trotzdem ausmachen. [pause] Und das ist jetzt alles mur Spekulation. Mit dem Verstärker, [pause] ob der Rechner von Teilnehmer B viel verbraucht, [pause] kein Ahnung, ob das so ist oder ob das nicht so ist. Ich weiß es wirklich nicht."

Gegen Ende des Workshops einigen sich die Teilnehmer darauf, die grau gefärbten Verbräuche in den Nebenzeiten zu minimieren. Die Teilnehmer bekommen die erwähnten Messuhren ausgehändigt, in ihrer Funktion erläutert und verlassen den Workshop. Die Workshopleitung erinnert nochmals daran, dass die Messung weiter durchgeführt wird, um evt. eintretende Effekte nachvollziehbar zu machen. 
Verteilung des Stromverbrauchs auf Hauptarbeits- und Nebenzeiten (nach dem Workshop)

Hauptzeit $\boxminus$ Nebenzeit

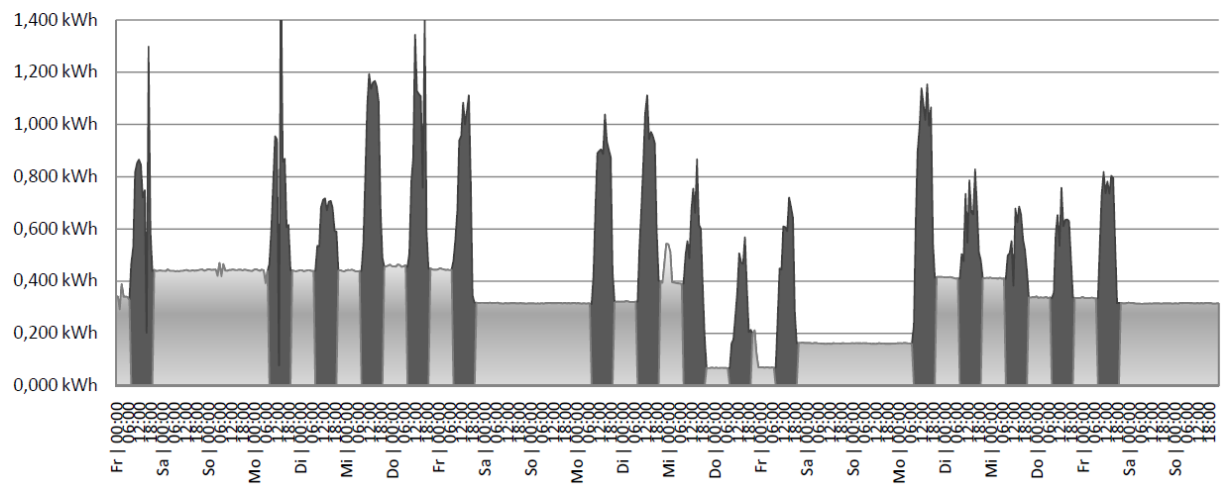

Abbildung 2: Lastgang Zeitraum: 3 Wochen nach dem partizipativen Auswertungsworkshop

\subsection{Lastgang unmittelbar nach dem Workshop}

Unter den veränderten Bedingungen wurde die gleiche Messung wie vor dem Workshop auch drei Wochen nach dem Workshop durchgeführt. Wie in Abbildung 2 deutlich wird, ist vor allem die Grundlast am zweiten und dritten Wochenende deutlich zurückgegangen. Es stellte sich bei der Verwendung der Messuhren durch die Teilnehmer der Studie heraus, dass der im Workshop mehrfach erwähnte „Mac Pro“ (ein Rechner, der überwiegend zum Videoschnitt und auch als FileServer genutzt wird) erheblich zur Grundlast beiträgt (312W Leistungsaufnahme ohne Monitore) und bisher nicht zum Arbeitsende oder am Wochenende abgeschaltet wurde. Ebenso wurde das gemeinsam genutzte Smartboard vollständig vom Netz getrennt, da es eher selten genutzt wird und einen Stand-by-Verbrauch von 38W aufweist. Zusätzlich haben sich die Workshopteilnehmer nach dem Workshop darauf verständigt, die gemeinsam genutzte Stereoanlage abends und am Wochenende vom Netz zu trennen. Stellt man den Stromverbrauch der drei Wochen vor dem Workshop und den der drei Wochen nach dem Workshop gegenüber, konnte der Verbrauch in den Nebenzeiten (19:30-7:30 Uhr) von durchschnittlich 0,288 kWh pro Stunde auf 0,217 kWh gesenkt werden. Dies entspricht einer Einsparung von 24,9\%.

\subsection{Lastgang in der langfristigen Perspektive}

Um die langfristigen Effekte nachvollziehbar zu halten, wurde die Messung des Gesamtstromverbrauchs der betreffenden Büroräume weiter fortgeführt. In Abbildung 3 ist der Lastgang über etwas mehr als 4 Wochen ca. 11/2 Monate nach dem durchgeführten Workshop dargestellt. Es wird deutlich, dass der Verbrauch zunächst in Nebenzeiten und am Wochenende etwas niedriger ausfällt, jedoch nicht 
Verteilung des Stromverbrauchs auf Hauptarbeits- und Nebenzeiten (langfristig)

घhauptzeit $\boxminus$ Nebenzeit

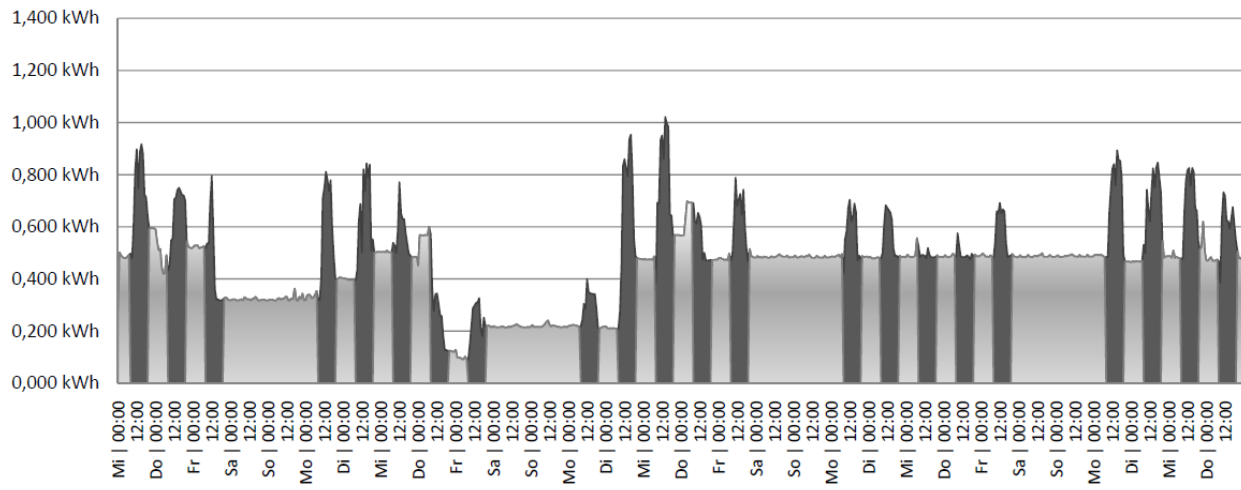

Abbildung 3: Lastgang langfristige Entwicklung nach dem Workshop

mehr solch starke Effekte wie in Abbildung $2 \mathrm{zu}$ beobachten sind. Im weiteren Verlauf steigen die Verbräuche in den Nebenzeiten wieder auf das Maß an, wie es bereits vor dem Workshop vorgefunden wurde.

Betrachtet man die genauen Verbräuche der Nebenzeiten, ergibt sich nun ein durchschnittlicher Verbrauch pro Stunde von 0,264 kWh. Dieser Wert liegt nun nur noch 8,4\% unter den durchschnittlichen Verbräuchen in den drei Wochen vor dem Workshop. Die Verbrauchsdaten der Nebenzeiten über alle drei betrachteten Phasen ergeben den in Abbildung 4 dargestellten Verlauf. Die dunkelgraue Kurve stellt den Trend (polynomisch) dar.

Das in dem Workshop bereitgestellte Feedback über den Stromverbrauch der Vergangenheit und die im Workshop gemeinsam erarbeiteten Ziele werden demnach zunächst in Angriff genommen und erzielen einen signifikanten Effekt, nämlich 24,9 \% Einsparungen in den Nebenzeiten. Ein weiteres Feedback über den Verbrauch wurde in der darauffolgenden Phase jedoch nicht gegeben, auch wurde kein weiterer Workshop durchgeführt. Gleichzeitig lässt der Einsparungseffekt deutlich nach.

\section{Zusammenfassung und Ausblick}

Die Studie hat gezeigt, dass durch die Verwendung von Messtechnologien verbunden mit einem daran gekoppelten Auswertungsworkshop zur Identifikation von Einsparpotentialen positive Effekte in Bezug auf den Energiekonsum in den gemessenen Büroräumen festellbar waren. Als wesentliches Ergebnis der Studie hat sich gezeigt, dass Aushandlungsprozesse auf sozialer Ebene ohne technische Intervention zu einer Verringerung des Stromverbrauchs geführt haben. Die Mitarbeiter in den untersuchten Büroräumen haben in Bezug auf die von Ihnen verwendeten elektrischen Geräten am Arbeitsplatz ohne eine gezielte Beeinflussung der Work- 
shopmoderatoren ein kollektives Problembewusstsein aufgebaut. Es wurde ersichtlich, dass Potentiale und Motivationen zur Verringerung des Energieverbrauchs bei den Akteuren latent vorhanden waren und im Rahmen des Workshops gehoben wurde. Dabei haben die Teilnehmer der Studie im Sinne des „Double-LoopLearnings" (Argyris 1982) ihre handlungsleitenden Logik expliziert und in Bezug auf den Umgang mit den vorhandenen elektrischen Geräten kollektiv reflektiert.

Die Auswertung des empirischen Materials hat gezeigt, dass sich das kollektiv gebildete Problembewusstsein als Handlungstreiber besonders wirksam herausgestellt hat, da das Verhalten des einzelnen stetig von der Gruppe auf die im Workshop diskutierten Zielen mit reflektiert wurden. Es wurde deutlich, dass die Kontrolleffekte der Gruppe ein nachhaltig energieeffizientes Verhalten des einzelnen Mitarbeiters gefördert hat und dass das individuelle Handlungsfeld auf Basis der diskutierten Verbrauchsdaten bestimmt wurde. Ferner ist zu unterstellen, dass sich die beobachteten Effekte ohne die Durchführung des Feedbackworkshops nicht eingestellt hätten.

Aus der durchgeführten Studie geht also hervor, dass detaillierte Informationen über Energiekonsum am Arbeitsplatz zu einem besseren Verständnis über die Verwendung der Ressource Elektrizität beitragen und der Schlüssel zur Identifikation von Einsparungspotenzialen sind. Liegen diese Informationen vor, ist es gleichermaßen wichtig, die Messdaten verständlich und bedarfsgerecht aufzubereiten und über angemessene Kanäle an den Nutzer von elektrischen Geräten zurück zu spiegeln. Ansätze zur Schaffung eines energieeffizienten Arbeitsplatzes durch mehr Energietransparenz und die Verwendung von intelligenter Messtechnologie stehen jedoch noch am Anfang. Dabei ist gleichzeitig ein Trend zur Verbreitung von intelligenter Messtechnologie zu beobachten. Möchte man Büroarbeitsplätze nachhaltig energieeffizient gestalten, ist es wichtig, bei der Gestaltung und Konzeption

Trend der Stromverbräuche in den Nebenzeiten (vor | nach | langfrist)

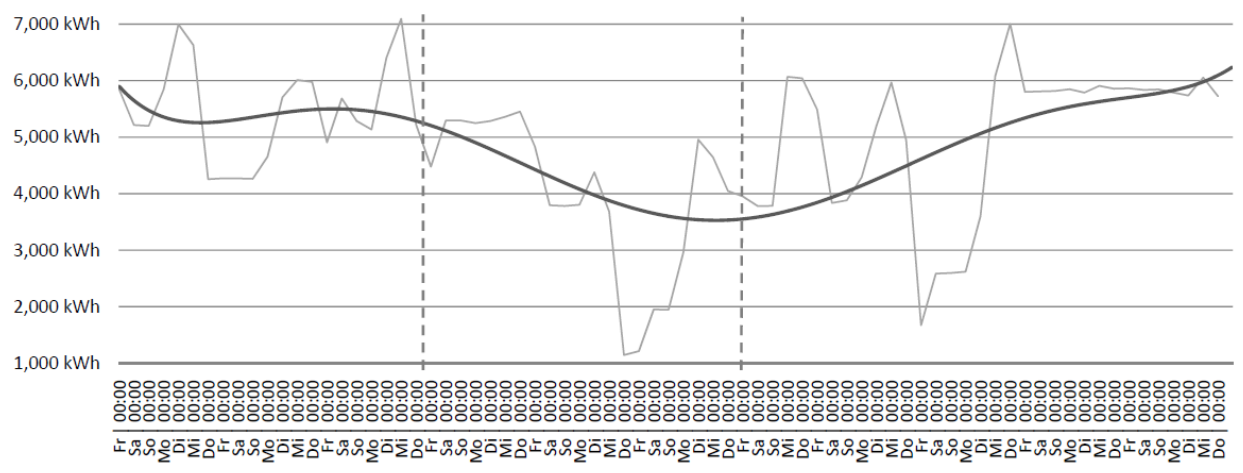

- Tagessummen Nebenzeiten nivelliert —Trend (polynomisch)

\section{Abbildung 4: Trend der Tagessummen der Verbräuche in Nebenzeiten über die Zeit: vor | nach dem Workshop und langfristige Entwicklung}


von Feedback-Systemen auch die soziale Dimension von Smart Metering zu berücksichtigen.

Basierend auf den gewonnenen Einsichten und Erkenntnissen müssen in weiterführenden Forschungsansätzen die Effekte von der Sichtbarmachung von Energieverbrauchsdaten am Arbeitsplatz näher untersucht werden. Geplant ist die Granularität der intelligenten Messtechnologie zu erhöhen und die zeitlichen Abstände zwischen Energiekonsum und Feedback zu verringern. Sich einstellende Phänomene sollen dabei in-situ beobachtet werden.

\section{Literatur}

Argyris C (1982) Organizational learning and management information systems. In: ACM SIGMIS Database. 3-11, 13 (2-3).

Chetty M, Brush AB, Meyers BR, Johns P (2009) It's not easy being green: understanding home computer power management. In: Proceedings of the 27th international conference on Human factors in computing systems. New York, NY: ACM 1033-1042.

Chetty M, Tran D, Grinter RE (2008) Getting to green: understanding resource consumption in the home. In: UbiComp '08: Proceedings of the 10th international conference on Ubiquitous computing. New York, NY, USA: ACM 242-251.

Darby S (2001) Making it obvious: designing feedback into energy consumption. In: Proceedings of the 2nd International Conference on Energy Efficiency in Household Appliances and Lighting. Berlin Heidelberg New York: Springer 685-696.

Darby S (2006) The effectiveness of feedback on energy consumption. A review for DEFRA of the literature on metering, billing and direct displays. Oxford, UK.

dena (2007) Deutschen Energie-Agentur GmbH (dena): Initiative

EnergieEffizienz. Der energieeffiziente Büroarbeitsplatz. 21.03.2007.

dena (2007) Hemmnisse für Energieeffizienz in Unternehmen. Hamburg

Dillahunt T, Mankoff J, Paulos E, Fussell S (2009) It's not all about "Green": energy use in low-income communities. In: ACM International Conference Proceeding Series. 9.

Hall A (2007) Tätigkeiten und berufliche Anforderungen in wissensintensiven Berufen. Studien zum deutschen Innovationssystem. Bonn.. 